\title{
SEDAÇÃO CONSCIENTE EM ODONTOLOGIA
}

\author{
CONSCIOUS SEDATION IN DENTISTRY
}

\author{
Cristiano Gaujac* \\ Harim Tavares dos Santos * \\ Moisés dos Santos Garção ** \\ Joéliton da Silva Júnior ${ }^{* * *}$ \\ José Renato Moraes Carvalho Barreto Brandão "* \\ Tarcisio Barbosa da Silva "*
}

\section{RESUMO}

O óxido nitroso, primeiro gás inalatório utilizado na sedação consciente, foi indentificado por Joseph Priestley. Esse composto ficou conhecido como gás hilariante, porém só em 1844 teve sua primeira aplicação em pacientes. Tem como características farmacológicas metabolizaçáo lenta no organismo, início e término de ação rápidos, mas alcançando concentrações cerebrais eficientes. Sua técnica baseia-se na administração de $\mathrm{O} 2$ seguida de $\mathrm{N} 2 \mathrm{O}$, até alcançar o nível de sedação ideal. Essas técnicas são indicadas para pacientes ansiosos, tensos e situações em que não existam contra-indicaçóes, porém essas são técnicas apenas relativas e não absolutas, e estão relacionadas principalmente ao risco de hipóxia, aumento de volume ou pressão em espaços fechados, alteraçóes hematológicas e neurológicas. Os benzodiazepínicos servem também como método de sedação consciente devido a sua ação ansiolítica: vale destacar o midazolam, diazepam, alprazolam, triazolam e lorazepam. Os efeitos colaterais são variados abrangendo degeneração da medula óssea, náuseas, teratogênese, entre outros. $\mathrm{O}$ objetivo deste trabalho é realizar uma revisão da literatura para mostrar a importância da sedação consciente nos procedimentos odontológicos.

DESCRITORES: Oxido nitroso • Farmacologia • Sedação consciente.

\section{ABSTRACT}

Nitrous oxide, the first inhaled gas used in conscious sedation, was identified by Joseph Priestley. This compound was known as laughing gas, but only in 1844 had its first application in patients. Its pharmacological characteristics are don't be metabolized in the body, beginning and ending of fast action, but reaching effi cient concentrations in the brain. Its technique is based on the administration of $\mathrm{O} 2$ followed by $\mathrm{N} 2 \mathrm{O}$, to achieve the optimal level of sedation. Such techniques are indicated for anxious and tense patients, and situations that have no contraindications, but these are only relative and not absolute, and are due mainly to the risk of hypoxia, volume or pressure increase in enclosed spaces, hematological and neurological changes. The benzodiazepines also serve as a conscious sedation method because of their anxiolytic action, highlight the valley midazolam, diazepam, alprazolam, triazolam and lorazepam. Side effects are varied covering bone marrow degeneration, nausea, teratogenesis, among others. The aim of this article is to realize a review in order to show the value of conscious sedation to dental procedures.

DESCRIPTORS: Nitrous oxide • Pharmacology • Conscious sedation.

\footnotetext{
* Professor do Departamento de Odontologia da Universidade Federal de Sergipe, e-mail: cgaujac@gmail.com.br

** Acadêmico do curso de odontologia da Universidade Federal de Sergipe - UFS, e-mail: manoharim@hotmail.com

** Acadêmico do curso de odontologia da Universidade Federal de Sergipe - UFS, e-mail: msg_odonto-ufs@hotmail.com

** Acadêmico do curso de odontologia da Universidade Federal de Sergipe - UFS, e-mail: jr.joeliton@hotmail.com

** Acadêmico do curso de odontologia da Universidade Federal de Sergipe - UFS, e-mail: renatinhu_brandaum@hotmail.com

** Acadêmico do curso de odontologia da Universidade Federal de Sergipe - UFS, e-mail: tarcisio-silva@hotmail.com
} 


\section{1-REVISÃO DA LITERATURA}

\section{1 - História do Óxido Nitroso (N2O)}

O óxido nitroso foi chamado de gás hilariante, gás do riso, dióxido de nitrogênio e protóxido de azoto, descoberto pelo químico inglês Joseph Priestley (1733-1804), que foi o primeiro a identificar e isolar o óxido nitroso, desconhecendo as propriedades analgésicas desse gás e sem imaginar a importância que o N2O teria na medicina futuramente (Rang $e t a l^{40} ., 2004$ ).

As experiências com o óxido nitroso começaram quando Humphrey Davy (1778 - 1829), um aprendiz de farmácia, no ano de 1798 , foi acometido por uma forte odontalgia causada pela erupção do $3^{\text {a }}$ molar e inalou o gás a $100 \%$, verificando um alívio na dor, desejo de rir e uma sensação agradável de euforia $\left(\right.$ Nocite $\left.^{34}, 1993\right)$.

O cirurgião-dentista Horace Wells $(1789$ - 1869) interessou-se pelas propriedades analgésicas do N2O, aperfeiçoando a técnica, fazendo com que as cirurgias fossem executadas sem dor. Ele também difundiu o uso do gás entre vários dentistas da época (Falqueiro $\left.{ }^{18}, 2004\right)$.

Com o uso progressivo, na década de 40, do éter e Clorofórmio, o uso do óxido nitroso entrou em desuso, mas logo voltou à popularidade por ser indicado na redução da ansiedade. Cerca de 56\% dos dentistas e $85 \%$ dos cirurgióes buco-maxilo-faciais e odontopediatras, nos EUA, fazem o uso do gás $\mathrm{N} 2 \mathrm{O} / \mathrm{O} 2$, para analgesia inalatória.

No Brasil, os benefícios físicos e psicológicos da técnica de sedação consciente com o óxido nitroso/oxigênio já são usadas em consultórios e não se restringem à área odontológica. Embora antiga e muito difundida nos EUA e Europa, sua utilização ainda gera polêmica entre os profissionais da saúde brasileiros, devido à falta de informação, pois muitas universidades não se dedicam ao assunto, dificultando o acesso do dentista às técnicas de sedação. As previsões de mercado apontam para um aumento no consumo desse produto num futuro próximo (Cavalcante e Nunes ${ }^{10}, 2003$ ).

\section{2 - Mecanismo de Açáo}

O óxido nitroso atua no sistema nervoso, com mecanismo de ação ainda não elucidado, promovendo uma leve depressão da córtex cerebral, e de foma diferente dos benzodiazepínicos que atuam a nível de bulbo, não deprime o centro respiratório, mantendo o reflexo laríngeo. Tranquiliza o paciente de forma rápida e segura, diminuindo a sua sensibilidade à dor. $\mathrm{O}$ óxido nitroso possui propriedades analgésica e sedativas (Caldas e
$\left.\mathrm{Gamba}^{9}, 2004\right)$.

O uso do oxido nitroso aumenta o limiar de dor no periósteo, levando a crer que pequenos procedimentos cirúrgicos na gengiva e mucosa podem ser executados sem o uso de complementação anestésica (Everett ${ }^{17}$, 1971). Estímulos elétricos realizados no lábio inferior foram suportados com voltagem bem superior, quando o paciente está sob o efeito do óxido nitroso, em percentuais superiores a 50\% (Trieger $\left.{ }^{45}, 1994\right)$. O efeito analgésico da sedação com óxido nitroso é relevante apenas durante a cirurgia, sem efeito depois do procedimento (Ong et $a^{\beta 6}$., 2004).

Sua pouca solubilidade no sangue e nos tecidos prover propriedades farmacocinéticas adequadas a um agente inalatório, principalmente como coadjuvante, tomando-se como parâmetro que sua captação e distribuição são muito rápidas, assim como sua eliminação (Eger e Larson $^{16}$, 1964; Thornton et al ${ }^{44}$., 1973).

A velocidade de transporte através das membranas biológicas é elevada, levando a eficazes efeitos específicos e globais no sistema nervoso central. Desse modo, em cerca de 5 minutos ele alcança os níveis ideais de sedação, ficando o paciente, então, tranquilo, responsivo e relaxado para o procedimento que será realizado. No término, dentro de minutos, ele estará livre para a execução de suas atividades rotineiras (Malamed ${ }^{30}$, 2003).

\section{3 - Indicaçóes e Contra-indicaçóes}

As complicaçóes do medo, da ansiedade e da fobia diante ao tratamento odontológico têm sido objeto de estudos há muito tempo. Esses fatores vêm sendo apontados, em pesquisas recentes, como responsáveis por um grande índice de doenças bucais e manifestações sistêmicas, que se tornaram um problema de saúde pública, apesar de todo o avanço dentro da Odontologia (Barbería et $a \bar{F}$., 2004). Ou seja, o estresse é um dos maiores problemas no tratamento odontológico, sendo necessário, portanto, seu controle (Malamed $\left.{ }^{30}, 2003\right)$.

Um dos métodos que o profissional possui para o controle desse medo é a ultilização de anestesia por via inalatória, uma técnica abrangente indicada em situaçóes como pacientes ASA I e ASA II para atendimento eletivo e ASA III para atendimento de emergências $\left(\right.$ Malamed $^{30}$, 2003). Entretanto, o paciente ASA III precisa de hospitalização, devendo-se observar se existe comprometimento no sistema respiratório ou cardíaco, pois pode favorecer a dificuldade de oxigenação e perfusão sanguínea em sistema nervoso central e depressão em sistema respiratório 
(Golnick e Mandeville 22 , 2002).

O uso do óxido nitroso é útil também em pacientes ansiosos, portadores de doenças cardiovasculares, doenças respiratórias não obstrutivas, doenças hepáticas, oncológicas, renais, com distúrbios neurológicos, distúrbios endócrinos incluindo diabetes, pacientes alérgicos, exceto ao látex em função das antigas máscaras que eram feitas desse material (as atuais são "látex free"); pacientes portadores de desordens nutricionais, leucêmicos e anêmicos (Sonis et alt3., 1996).

O uso desse gás pode ser utilizado para tratamento rotineiro de pacientes odontopediátricos, pois é seguro e eficaz, influenciando decisivamente no comportamento, diminuindo os níveis de ansiedade na sequência de consultas, e é eficaz também para pacientes portadores de doenças crônicas (Veerkamp $\left.{ }^{46}, 1995\right)$. Sabe-se, ainda, que o controle da dor em pacientes com insuficiência coronariana é a segunda maior indicação (Parbrook ${ }^{38}$, 1968).

Existem contra-indicaçóes sistêmicas e locais. As primeiras são infecçóes agudas das vias respiratórias superiores; doença pulmonar obstrutiva crônica (DPOC), doenças sistêmicas severas, pacientes psicóticos, portadores de miastenia gravis, esclerose múltipla, hérnia diafragmática, desordens decorrentes da deficiência B12 e gravidez. Já as contra-indicaçóes locais são procedimentos que poderão interferir com a máscara nasal; Há necessidade de se manter livre a via aérea, como por exemplo, drenagem de abscesso intra-oral, e em respiradores bucais (Berge et al ., 2001).

As contra-indicaçôes do N2O devem-se principalmente ao risco de hipóxia, aumento de volume ou de pressão em espaços fechados (pneumotórax), alteraçôes hematológicas ou neurológicas segundo Rang et at ${ }^{40}$, 2004.

\section{4 - Técnica de Utilização da Analgesia Inalató- ria}

O sucesso da técnica está na análise da resposta do paciente. Antes do primeiro uso no dia, devem ser verificados: oferta de oxigênio, quantidade de $\mathrm{N} 2 \mathrm{O}$, funcionamento do fluxômetro, funcionamento do aspirador, disponibilidade de fármacos. Para compreensão da analgesia, deve-se ter conhecimento de anestesia geral do Plano de Guedel, que analisou diversos sinais e sintomas e verificou mudanças em períodos de progressão anestésica: analgesia, delírio, analgesia cirúrgica e paralisia respiratória (Falqueiro $\left.{ }^{18}, 2004\right)$.
A técnica de sedação inalatória se resume da seguinte forma: Teste de Trieger; de acordo com o perfil facial do paciente é escolhida a máscara nasal; regulação do volu$\mathrm{me} /$ minuto em L/min; pré-oxigenaçáo (variando em 3 minutos); elevação da taxa de N2O; pós-oxigenação (variando em 5 minutos); realização do teste de Trieger novamente, para se analisarem os resultados, verificando-se a possibilidade de alta do paciente (Malamed $\left.{ }^{31}, 2005\right)$.

Determinado o fluxo preferível e verificado o bom ajustamento da máscara no paciente, começa-se a liberaçáo do óxido nitroso em incrementos, geralmente de $10 \%$ a cada minuto, até que seja verificado um bom nível de sedação, com relaxamento e bem-estar do paciente. A determinação da sedação ideal é subjetiva, varia de paciente para paciente, mas sempre limitado ao máximo de 70\% de N2O. É importante ressaltar que, mesmo na utilização da máxima concentração, é também ofertada uma concentração extra de oxigênio, aproximadamente $50 \%$ a mais do que o ar atmosférico (Ramacciato et $a^{\beta}$, 2004).

De acordo com a necessidade do procedimento odontológico, é escolhido o volume da bolsa respiratória. A máscara facial selecionada é a que mais se adéqua à anatomia do paciente. $\mathrm{O}$ ajuste do fluxo de oxigênio varia em: 6 a $7 \mathrm{~L} / \mathrm{min}$ para adulto e 4 a $5 \mathrm{~L} / \mathrm{min}$ para crianças. A aparelhagem é ajustada para liberar $100 \%$ de oxigênio. Colocar a máscara facial no paciente. A ajuda do paciente para adequar a máscara o mais firme possível e confortável é de grande valia. O paciente pode ajustar a máscara durante o decorrer do procedimento. Para assegurar um ajuste perfeito, ajuste os tubos condutores por trás da cabeça do paciente. Uma adequação inapropriada causa vazamento de gás, o que contaminará o meio à volta. Colocar uma gaze dobrada entre o nariz e a máscara é de grande proveito (Falqueiro ${ }^{18}$, 2004).

Observação da bolsa respiratória - ela é um bom indicador de fluxo adequado. Se a bolsa estiver inflando em demasia, diminua o L/min, mas se ela continuar inflando como um baláo é possível que tenha ocorrido uma obstruçáo do fluxo de alguma forma. No início da perda de potência da bolsa, deve-se elevar o volume. Sugerir ao paciente: não respire pela boca; náo conversar durante a analgesia. Evite que o paciente fale para não contaminar o meio em volta e não diminuir o efeito (Falqueiro ${ }^{18}$, 2004).

\section{5 - Benzodiazepínicos}

Além da sedação consciente promovida pelo óxido 
nitroso, há outro método que pode chegar a esse fim: a utilização dos benzodiazepínicos, que visa diminuir a ansiedade do paciente ao tratamento odontológico.

A ansiedade e o medo do tratamento odontológico ainda persistem em boa parte da população, sendo gerados por fatores como os sons e vibraçóes dos instrumentos rotatórios, movimentos bruscos do profissional, relatos de parentes ou amigos que tiveram experiências negativas em consultas anteriores, além da anestesia local, relatada como o procedimento mais estressador no consultório odontológico. $\mathrm{O}$ medo dos procedimentos odontológicos está presente em grande parcela da sociedade, sendo que mais de $40 \%$ das pessoas evita procurar a assistência ao profissional devido ao medo e ansiedade ( Ayer et al ${ }^{2}$., 1983; Aeschliman et al'., 2003).

Os benzodiazepínicos estão entre as drogas mais largamente prescritas e empregadas em todo o mundo, constituindo a principal modalidade terapêutica para o tratamento de desordens relacionadas à ansiedade (Cogo et $a l^{12}$., 2006).

Essas drogas se ligam a receptores específicos em estruturas do sistema nervoso central (SNC), como o Sistema Límbico; facilitando a ação do ácido gama-amino butírico (GABA), o neurotransmissor inibitório primário do SNC. A ativação do receptor GABA induz a abertura dos canais de cloreto (cl-) da membrana dos neurônios, aumentando o influxo do ânion para dentro das células, o que resulta, em última análise, na diminuição da propagaçáo de impulsos excitatórios (Loeffler ${ }^{28}$, 1992; Rang et $a l^{40}$., 2004).

Essas drogas podem ser classificadas de acordo com o tempo de duração de sua ação ansiolítica ou sedativa:

Diazepam: Considerado um agente de longa duração de ação com meia-vida de eliminação entre 24 e 72 horas, contrastando com outras drogas do grupo. Apesar dos efeitos clínicos desaparecerem em 2 a 3 horas, a sonolência e o prejuízo na função psicomotora podem persistir devido à produção de metabólitos ativos (Loeffler $\left.^{28}, 1992\right)$.

Lorazepam: Difere do diazepam por não produzir metabólitos ativos, e o término de seus efeitos é observado após 6 a 8 horas. Por essa razão e pelo fato de dificilmente produzir efeitos paradoxais, é considerado por alguns autores como o agente ideal para a sedação consciente de pacientes idosos (Matear e Clarke ${ }^{32}$, 1999; Goodchild et al ${ }^{3}$., 2003).

Alprazolam: Suas maiores concentrações plasmáticas são obtidas de 1 a 2 horas após sua administração, com duração de ação de 12 a 15 horas (Matear e Clarke ${ }^{32}$, 1999).

Midazolam: Apresenta propriedades ansiolíticas, miorrelaxantes, anticonvulsionantes e psicosedativas. Possui eliminação rápida, e ocorre através da degradação no fígado, independente da via de administração (Fukuta $e t a l^{11}$., 1994; Juaréz-Lopez et $a l^{27}$., 1998).

Triazolam: Benzodiazepínico de curta duração, comparável ao lorazepam como medicação pré-anestésica, mas com início de ação mais rápido (30 a 60 minutos) e menor tempo de recuperação, (em torno de 2 a 4 horas) (Berthold $e t a l$., 1993; Dionne et $a l^{14}$., 2002; Goodchild et $\left.a l^{3} ., 2003\right)$.

\section{6 - Efeitos Colaterais}

Por causa de sua baixa solubilidade, o N2O pode provocar efeitos de aumento da pressão e/ou volume em cavidades. $\mathrm{O}$ aumento de pneumotórax, embolismo aéreo, aumento da pressão no ouvido médio, aumento da pressão nos seios da face, distensão de alças intestinais, aumento de bolhas de gás no espaço epidural e consequente compressão nervosa (Maino et $a l^{29}$., 2005).

$\mathrm{O}$ óxido nitroso pode interferir no metabolismo da vitamina B12 e pode causar degeneração da medula espinhal. $\mathrm{O}$ mecanismo é devido à inativação da vitamina B12 que leva a imparidade na síntese de metionina (Filippo e Holder ${ }^{19}$, 1993). Assim, em indivíduos expostos a longo prazo ao $\mathrm{N} 2 \mathrm{O}$, existe a possibilidade de ocorrência de alteraçốes megaoblásticas (Banks et al ${ }^{4}$., 1968).

Inativando-se a metionina sintetase, ocorre diminuição de metionina e do tetrahidrofolato. A metionina tem papel como doadora de um grande número de reações de metilação, como a mielinização. O efeito do N2O na produção de metionina pode acarretar uma mielinização modificada e uma degeneração subaguda da medula (Nunn $\left.{ }^{35}, 1987\right)$.

São necessárias várias horas ou até mesmo dias sob exposição do óxido nitroso para que seja detectado algum tipo de teratogênese. Essa observação deve ser direcionada aos pacientes expostos por várias horas ao $\mathrm{N} 2 \mathrm{O}$ em altas concentraçóes e aos profissionais que trabalham diretamente com esse produto (Fink et al20., 1967).

Outros efeitos colaterais são: produção de estímulo simpático que aumenta os efeitos indesejáveis dos vasoconstritores, levando a picos hipertensivos; aumento da pressão no ouvido médio que pode levar a efeitos pósoperatórios adversos na audição; aumentos indesejados na pressão intraocular (Negreiros $\left.{ }^{34}, 2004\right)$. 
Acredita-se que ocorra maior incidência de náuseas e vômitos no pós-operatório com o uso de óxido nitroso. Estima-se que omitindo-se o oóxido nitroso da técnica anestésica se reduz o risco desses dois efeitos advervos em aproximadamente $28 \%$ (Divatia ${ }^{15}, 1996$ ).

Em relação aos benzodiazepínicos, eles mostram poucas reações adversas nos procedimentos odontológicos. Mas alguns pacientes apresentam como efeitos colaterais sonolência, tontura, cefaleia, náusea, secura na boca, glossite, distúrbios gastrintestinais, astenia, apatia, lassidão, e parestesia. Alguns derivados benzodiazepínicos podem ainda eventualmente levar a efeitos paradoxais, como insônia, hiperessitabilidade, agitação e hostilidade (Goodman e Gilman ${ }^{24}$, 1996).

No sistema respiratório, os benzodiazepínicos podem diminuir o volume de ar corrente e a frequência respiratória, justificando a recomendaçáo de serem empregados com precaução em pacientes portadores de enfermidade broncopulmonar obstrutiva ou com insuficiência respiratória (Salazar $\left.{ }^{42}, 1999\right)$.

\section{2 - DISCUSSÃO}

A sedação consciente na Odontologia é mais comumente aplicada através de benzodiazepínicos e pelo óxido nitroso. Este tem como vantagens perante os benzodiazepínicos o fato de ter início rápido, e pela dosagem ser obtida de forma incremental. Entretanto, para se conseguir autorização para o uso da técnica, obtenção do equipamento e acessórios existe a necessidade de maiores gastos do profissional (Cogo et al' ${ }^{12}$., 2006).

Devido à sua baixa afinidade lipídica ocorre uma rápida absorção, e desse modo a velocidade de transporte através das membranas biológicas é considerável, levando a eficazes efeitos específicos e globais no sistema nervoso central. Assim, sua ação no organismo é rápida, fato que contribui para sua baixa toxicidade e baixo índice de complicaçóes no ato do atendimento Malamed $^{30}$, 2003).

Episódios de esquecimento, causados pelos benzodiazepínicos, dos fatos a partir de um momento estabelecido como referência são definidos como amnésia anterógrada. Esse tipo de amnésia é vista como benéfico para muitos odontólogos, porém não é almejado por outros, já que o paciente pode apresentar dificuldades em recorda-se dos cuidados pós-operatórios no caso de um procedimento cirúrgico mais invasivo (Blin et al ${ }^{\beta}$., 2001)

Analisando-se o sistema cardiovascular, os benzodiazepínicos possuem alguns efeitos benéficos, ligados à diminuição da pré e pós carga, devido à ação simpatolítica. Eles diminuem o consumo de oxigênio do miocárdio, mas associados a doses elevadas de opioides podem levar a uma importante depressão miocárdica, especialmente em pacientes com função ventricular comprometida (Howie ${ }^{25}$, 1997).

Os benzodiazepínicos apresentam poucos acontecimentos de efeitos adversos e toxicidade, principalmente em tratamentos de curta duração, como é o caso do uso em Odontologia. Mas uma pequena porcentagem dos pacientes (principalmente crianças e idosos) pode apresentar um "efeito paradoxal", caracterizado por excitação, agressividade e irritabilidade, mesmo em baixas doses (Oreland ${ }^{37}$, 1988).

\section{3 - CONSIDERAÇÕES FINAIS}

A sedaçáo consciente mostra-se como um método válido que não abrange somente o uso de óxido nitroso, mas também de outros medicamentos como os benzodiazepínicos. Seu papel na Odontologia vem sendo consolidado com o tempo, sendo essencial que o cirurgião-dentista tenha o conhecimento de seus benefícios e malefícios para uma prática odontológica satisfatória. 


\section{REFERÊNCIAS}

1. Aeschliman SD, Blue MS, Williams KB, Cobb CM, MacNeill SR. A preliminary study on oxygen saturation levels of patients during periodontal surgery with and without oral conscious sedation using diazepam. J Periodontol 2003 Jul; 74(7):1056-9.

2. Ayer WA, Demoto PK, Gale EN, Joy EDJr, Melamed BG. Overcoming dental fear: strategies for its prevention and management. J Am Dent Assoc 1983 Jul; 107(1):18-27.

3. Axelsson G, Ahlborg G, Bodin L. Shift work, nitrous oxide exposure and spontaneous abortion among Swedish midwives. Occup Envir Med 1996 Jun; 53(6): 374-8.

4. Banks RGS, Henderson RJ, Pratt JM. Reactions of gases in solution. Part III. Some reactions of nitrous oxide with transition-metal complexes. J Chemi Soc 1968 Dec; 3(1): 2886-9.

5. Barbería E, Fernandéz-Frías C, Suárez-Clúa C, Saavedra $\mathrm{D}$. Analysis of anxiety variables in dental students. Int Dent J 2004 Dec; 54(6): 445-9

6. Berge TI. Nitrous oxide in dental surgery: Best Practice. Res Clin Anaesthesiol 2001 Sep; 15(6):477-89.

7. Berthold CW, Schneider A, Dionne R. Using triazolam to reduce dental anxiety. J Am Dent Assoc 1993 Nov; 124(11):58-64.

8. Blin O, Simon N, Jouve E, Habib M, Gayraud D, Durand A et al. Pharmacokinetic and pharmacodynamic analysis of sedative and amnesic effects of lorazepam in healthy volunteers. Clin Neur Ophar Macel 2001 Mar; 24(2):71-81.

9. Caldas LAF, Gamba, CG. A sedação consciente e sua importância no controle diário da dor, medo e ansiedade na clínica odontológica. Revista Naval 2004 ago; 51: 3.

10. Cavalcante SR, Nunes RR. Avaliação dos parâmetros derivados do encefalograma durante administração de diferentes concentrações de óxido nitroso. Rev Bras Anest 200353 1-8

11. Clark M, Brunick A. HandBook of Nitrous Oxide and Oxygen. $3^{\text {a }}$ ed. St Loius: Mosby, 2004.
12. Cogo K, Bergamaschi CC, Yatsuda R, Volpato MC, Andrade ED. Sedação Consciente Com Benzodiazepínicos Em Odontologia, Rev Odont da UNICID 2006 maio-ago; 18(2)181-8

13. Cohen EN, Gift HC, Brown BW, Greenfield W, Wu ML Jones TW, Whitcher CE, Driscoll EJ, Brodsky JB. Occupational disease in dentistry and chronic exposure to trace anesthetic gases. J Am Dent Assoc 1980 Jul; 101(1): 21-31.

14. Dionne RA, Phero JC, Becker DE. Management of pain and anxiety in the dental office. W.B. Saunders. 2002 Aug: 229.

15. Divatia JV, Vaidya JS, Badwe RA, Hawaldar RW. Omission of nitrous oxide during anesthesia reduces the incidence of postoperative nausea and vomiting: a meta-analysis. Anesthesiology 1996 Nov 85(5):10551062.

16. Eger EI Land, Larson Jr CP - Anaesthetic solubility in blood and tissues: values and significance. $\mathrm{BrJ}$ Anaest, 1964 Mar; 36:140-4.

17. Everet GB, Allen GD. Simultaneous evaluation of cardiorespiratory and analgesic: effects of nitrous oxide/oxygen inhalation analgesia. J Am Dent Assoc 1971 Jul; 83(1):129-33.

18. Falqueiro JM. Analgesia Inalatória por Óxido Nitroso/ Oxigênio. 1a ed. São Paulo: Santos; 2004.

19. Filippo TS, Holder WD. Neurologic degeneration associated with nitrous oxide anesthesia in patients with vitamin B12 deficiency. Arch Surg 1993 Dec; 128(12):1391-5.

20. Fink BR, Shephard TH, Blandau RJ. Teratogenic activity of nitrous oxide. Nature 1967 Apr; 214(5084): 146-8.

21. Fukuta O, Braham RL, Yanase H, Atsumi N, Kurosu $\mathrm{K}$. The sedative effect of intranasal midazolam admimistration in the dental treatment of patients with mental disabilities. Part 2 - Optimal concentration of intranasal midazolam. J Clin Pediatr Dent 1994 Summer;18(4):259-65.

22. Golnick JM, Mandeville M. Considerations for Monitoring Pediatric Sedations. J Mich Dent Assoc 2002 Jan; 84(1):34-6. 
23. Goodchild JH, Feck AS, Silverman MD. Anxiolysis in general dental practice. Dent Today 2003 Mar; 22(3)106-11.

24. Goodman LS, Gilman A. As bases farmacológicas da terapêutica.10. Ed. Rio de Janeiro: Mcgraw Hill; 1996.

25. Howie MB. Use of intravenous techniques in critically patients. In: WHITE PF. ed. Textbook of intravenous anesthesia. Wiliams \& Wilkins, Baltimore, cap. 25, p. 503- 513; 1997.

26. Iwata K, O’Keefe GB, Karanas A. Neurologic problems associated with nitrous oxide abuse in a nonhealthcare worker. Am J Med Sci 2001 Sep; 322(3): 173-4.

27. Juaréz-López L, Saavedra-García M, Ramírez-González G. Estudio comparativo entre dos esquemas de sedación en pacientes odontopediátricos. Bol Med Hosp Infant. 1998; Ene; 55

28. Loeffler PM. Oral benzodiazepines and conscious sedation: a review. J Oral Maxillofac Surg 1992 Sep; 50(9): 989-97.

29. Maino P, Dullenkopf A, Bernet V, Weiss M. Nitrous oxide diffusion into the cuffs of disposable laryngeal mask airways. Anaesthesia. 2005 Mar; 60(3):27882.

30. Malamed SF: Sedation a guide to patient management. $4^{a}$ ed. St Louis: Mosby, 2003 p.167-278.

31. Malamed SF. Manual de anestesia local. 5a ed. Rio de Janeiro: Elsevier; 2005.

32. Matear DW, Clarke D. Considerations for the use or oral sedation in the institutionalized geriatric patient during dental interventions: a review of the literature. Spec Care Dentist 1999 Mar-Apr; 19(2):56-63.

33. Medcenter Odontologia. $\mathrm{O}$ uso do óxido nitroso por odontologos. Disponível em: http:www.odontologia. com.br/artigos.asp?id=529 (2004)

34. Nocite JR. Óxido nitroso: Perspectivas para o ano 2000. Rev Bras Anestesiol 1993; 43: 157-8 35. Nunn JF. Clinical aspects of the interaction between nitrous oxide and vitamin B12. Br J Anaesth 1987 Jan; 59(1): 3-13.
36. Ong KS, Seymour DDS, Tan JM. A prospective randomized crossover of study of the preemptive analgesic effect of nitrous oxide in oral surgery. Oral Surg Oral Med Pathol Oral Radiol Endod 2004 Dec; 98(4):637-42.

37. Oreland L. The benzodiazepines. a pharmacological overview. Acta Anaesthesiol Scand. Suppl 1988; 88:13-6.

38. Parbrook GD - Therapeutic use of nitrous oxide: a review. BrJ Anaesth 1968 Jun; 366-72.

39. Ramacciato JC, Ranali J, Motta RHL. Biossegurança na sedação inalatória com Óxido Nitroso. Rev Assoc Paul Cirur Dent, 2004 mar-abr; 58(2): 374-8.

40. Rang HP, Dale MM, Ritter P. Farmacologia. $5^{a}$ ed. Rio de Janeiro: Guanabara Koogan; 2004.

41. Rowland AS, Baird DD, Shore DL, Weinberg CR, Savitz DA, Wilcox AJ. Nitrous oxide and spontaneous abortion in female dental assistants. $A m J$ Epid 1995 Mar 15; 141(6): 531-8.

42. Salazar A. Anestesia general y sedación en odontologia. Acta Odontol Venez Caracas 1999 May; 32(2):6774.

43. Sonis ST, Fang L, Fazio RC. Princípios e práticas de medicina oral. $2^{a}$ ed. Rio de Janeiro: GuanabaraKoogan; 1996. cap 51 p. 451-3.

44. Thornton JA, Fleming JS, Goldberg AD - Cardiovascular effects of $50 \%$ nitrous oxide and $50 \%$ oxygen mixture. Anaesthesia 1973 Sep; 28(5): 484-9.

45. Trieger N, Pain Control Year Book $2^{\circ}$ ed St Louis:Mosby; 1994.

46. Veerkamp JS, Gruythuysen RJ, Hoogstraten J, van Amerongen WE. Anxiety reduction with nitrous oxide: a permanent solution?. ASDC J Den Child 1995 Jan-Feb; 62(1):44-48.

Recebido em: 19/4/2009

Aceito em: 1/10/2009 